\title{
Enhanced recovery after surgery (ERAS) protocols in neonates should focus on the respiratory tract
}

\author{
Yuji Wakimoto $^{1}$ - Sathyaprasad Burjonrappa ${ }^{2}$
}

Accepted: 9 January 2019 / Published online: 2 February 2019

๑) Springer-Verlag GmbH Germany, part of Springer Nature 2019

\begin{abstract}
Background/purpose Enhanced recovery after surgery (ERAS) protocols have shown significant benefits in terms of patient outcomes and institutional cost savings in colorectal and bariatric surgery. This has not, however, been tried in the neonatal setting. One of the major barriers to developing ERAS protocols in the neonatal intensive care unit (NICU) is the oftenprolonged intubation of neonates after surgery. To this end, we evaluated our institutional data to determine if prolonged intubation post-operatively is associated with adverse events.

Methods This is a retrospective cohort study of neonates who were intubated for a surgical procedure from January 2012 to December 2016. Documented data included pre-operative intubation status, timing of post-operative extubation: immediate $(<24 \mathrm{~h})$ or delayed $(>24 \mathrm{~h})$, and adverse respiratory events. The Fisher exact test and Student's $t$ test were used to study differences amongst categorical and continuous variables, respectively.

Results 58 surgical procedures were identified, where the patient was intubated specifically for the surgical intervention, of which 28 were extubated immediately and 30 were extubated in a delayed fashion. The overall incidence of adverse respiratory events was increased in the delayed extubation group $(P=0.03)$.

Conclusions Healthcare providers should encourage early extubation after neonatal surgery. Consideration should be given to implementing ERAS protocols in NICUs.

Level of evidence Prognosis study—level II.
\end{abstract}

Keywords Enhanced recovery after surgery $\cdot$ Neonates $\cdot$ Post-operative extubation $\cdot$ Intubation $\cdot$ NICU

\section{Introduction}

Enhanced recovery after surgery (ERAS) protocols have shown significant benefits in terms of patient outcomes (decreased complication rates) and facility cost savings in adult colorectal and bariatric surgery. ERAS is also being developed in the pediatric surgery setting in conditions such as appendicitis and elective colorectal operations. In pediatric cardiac surgery, early $(<24 \mathrm{~h})$ extubation after surgery was associated with a lower mortality rate and a lower rate of re-intubation as compared with those who had delayed extubation (beyond $24 \mathrm{~h}$ ) [1]. The authors in the study noted

Sathyaprasad Burjonrappa

sburjonrappa@health.usf.edu

1 NYU-Winthrop University Hospital, 259 1st St, Mineola, NY 11501, USA

2 University of South Florida, 1 Tampa General Circle G441, Tampa, FL 33606, USA that the benefits of early extubation could be extended to neonates and babies having complex cardiac surgery such as transposition and anomalous pulmonary venous return. The benefit of early extubation in the pediatric cardiac surgery setting has translated to improved resource allocation by reducing intensive care unit stay and overall hospital length of stay $[2,3]$. Other than in the neonatal cardiac surgery setting, early extubation has not been attempted or reported after surgery in neonates $[4,5]$. Any attempt at instituting ERAS in the neonatal surgery setting will have to address the often-prolonged mechanical intubation after surgery.

Intubation and mechanical ventilation is the most definitive method of accessing and maintaining the airway in patients undergoing complex surgery. Complications of intubation and mechanical ventilation have been identified in both the immediate and delayed settings. Incorrect intubation, unplanned accidental extubation, ventilatorassociated atelectasis and pneumonia, hypoxemia, acidosis, bradycardia-apnea-desaturation, and extubation failure with 
subsequent need for re-intubation have all been identified as adverse events in the immediate phase [6]. Chronic consequences of prolonged intubation include neurological sequelae due to intra-ventricular hemorrhage (IVH) and bronchopulmonary dysplasia (BPD) [7, 8]. As a consequence of the increasing importance attributed to quality metrics, intubation days' being one of them, there has been an evolution in the management of respiratory support in the modern NICU with an emphasis on early extubation in premature infants and non-invasive measures of respiratory support $[9,10]$. This evolution in care affords an opportunity to study the consequences of early extubation that would likely be the first step to instituting ERAS in the NICU. The objective of this study was to evaluate the incidence of adverse respiratory events after delayed extubation following non-cardiac neonatal surgery.

\section{Materials and methods}

This was a single-site retrospective cohort study in NICU patients in a level IV regional neonatal center from January 2012 to December 2016 who underwent a surgical procedure. Patients selected for the study were intubated for the operation. NICU patients who received surgery, but were not intubated and used other modalities of respiratory support such as an LMA or a spinal anesthesia and patients, whose intubation status was not clearly marked by the data set were excluded from the study. Patients were also excluded from this study if they were intubated and had ventilatory support in the immediate days leading up to their surgical procedures, due to the possible interference of their preexisting respiratory conditions that was independent of the surgical intubation. A data sheet was used to record the gestational age, birthweight, type of procedure performed, pre-operative intubation status, the status of post-operative extubation, adverse respiratory events, and days spent in the hospital following the operation. For the purpose of this study, post-operative extubation status was defined as immediate extubation when patients were extubated within $24 \mathrm{~h}$ of the procedure, or delayed extubation when the intubation persisted for greater than $24 \mathrm{~h}$ after the procedure. Adverse respiratory events that were tabulated include: extubation failure and need for re-intubation, unplanned or accidental extubation, atelectasis/pneumonia, bradycardia/apnea/desaturation, acidosis, and death (Table 1). The primary outcome for the study was determination of in-hospital adverse events due to decision to keep the patient intubated beyond $24 \mathrm{~h}$ post-operatively, and secondary outcomes focused on the length of hospital stay beyond the surgery.

We used descriptive statistics to determine inter-quartile ranges. As this was a small data set, even a single outlier could significantly influence the mean. The ROUT method was used to identify and discount outliers from non-linear regression (GraphPad Software, LaJolla, CA, USA). Briefly, in the ROUT method, one initially fits a model to the data, where outliers have little impact. Subsequently, a new outlier detection method, based on the false discovery rate (FDR), is used to decide which data points are far enough from the prediction of the model so as to be called an outlier. Chisquare and Student's $t$ tests were used to evaluate categorical and continuous variables.

\section{Results}

There were 99 surgical procedures performed during this period in NICU patients. Of these, 33 of them were identified as patients who used airway measures alternative to intubation and mechanical ventilation. Another set of eight procedures were separated from the study, because these NICU patients were already intubated in the immediate days leading up to their surgical procedures. 58 remaining procedures were identified that fit the inclusion criteria for the study, of which 28 procedures were followed by immediate extubation and 30 procedures with delayed extubation (Tables 2, 3). From the clinical chart in the system, where the data were extracted from, the decision to keep neonatal patients intubated post-operatively was not always identified. In certain patients, the use of sedatives and narcotics such
Table 1 Complications from prolonged intubation

\begin{tabular}{llll}
\hline Extubation status & $\begin{array}{l}\text { Immediate extubation } \\
(<24 \mathrm{~h})\end{array}$ & $\begin{array}{l}\text { Delayed extubation } \\
(>24 \mathrm{~h})\end{array}$ & $p$ value \\
\hline Need for re-intubation & 28 & 30 & \\
Unplanned extubation & $1(3.6 \%)$ & $3(10 \%)$ & 0.33 \\
Atelectasis/pneumonia & $0(0 \%)$ & $2(6.7 \%)$ & 0.16 \\
Bradycardia, apnea or desaturation & $1(3.6 \%)$ & $4(13.3 \%)$ & 0.19 \\
Acidemia & $5(17.9 \%)$ & $7(23.3 \%)$ & 0.61 \\
Death & $2(7.1 \%)$ & $1(3.3 \%)$ & 0.51 \\
Total number of events & $0(0 \%)$ & $1(3.3 \%)$ & 0.33 \\
\hline
\end{tabular}









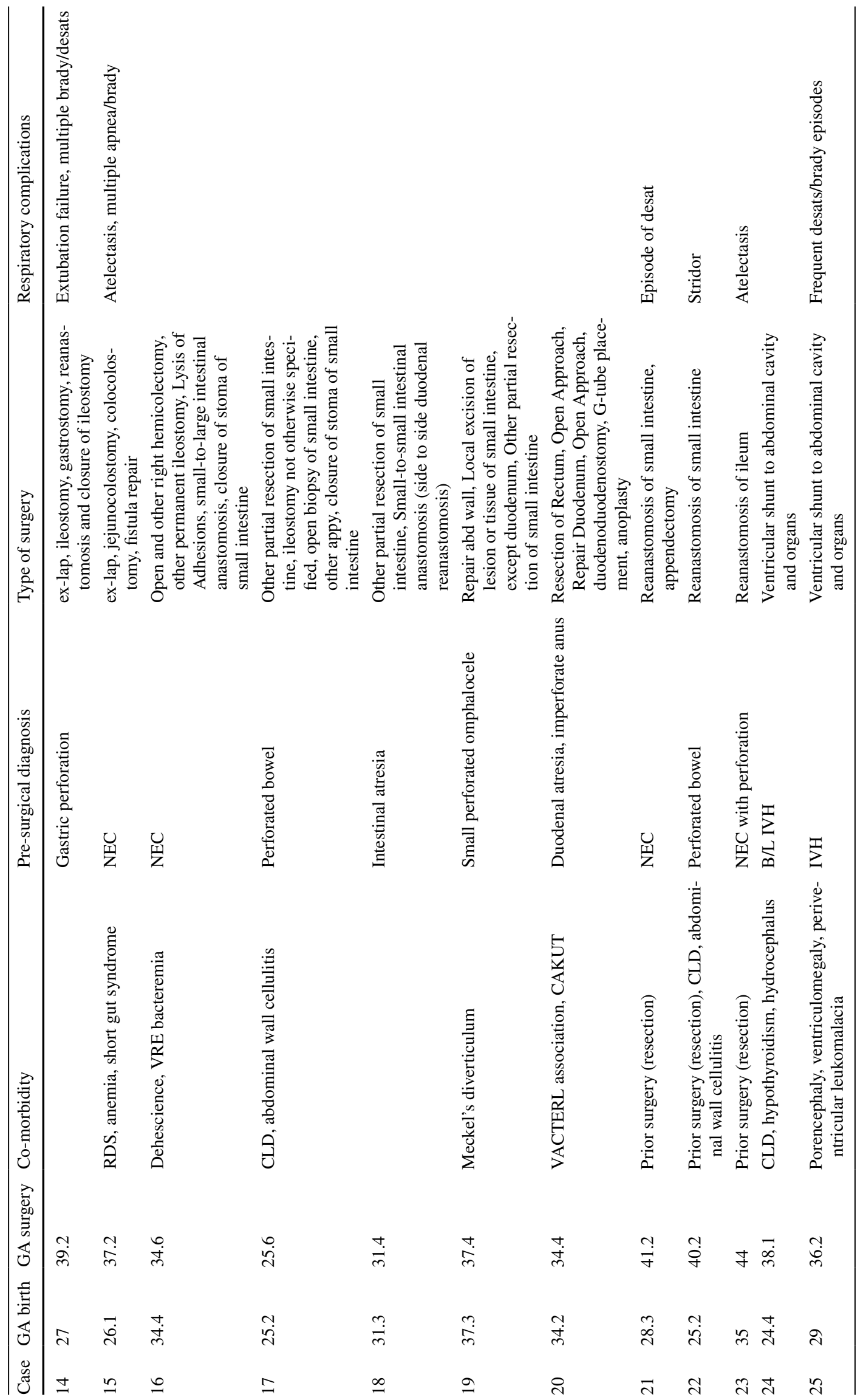




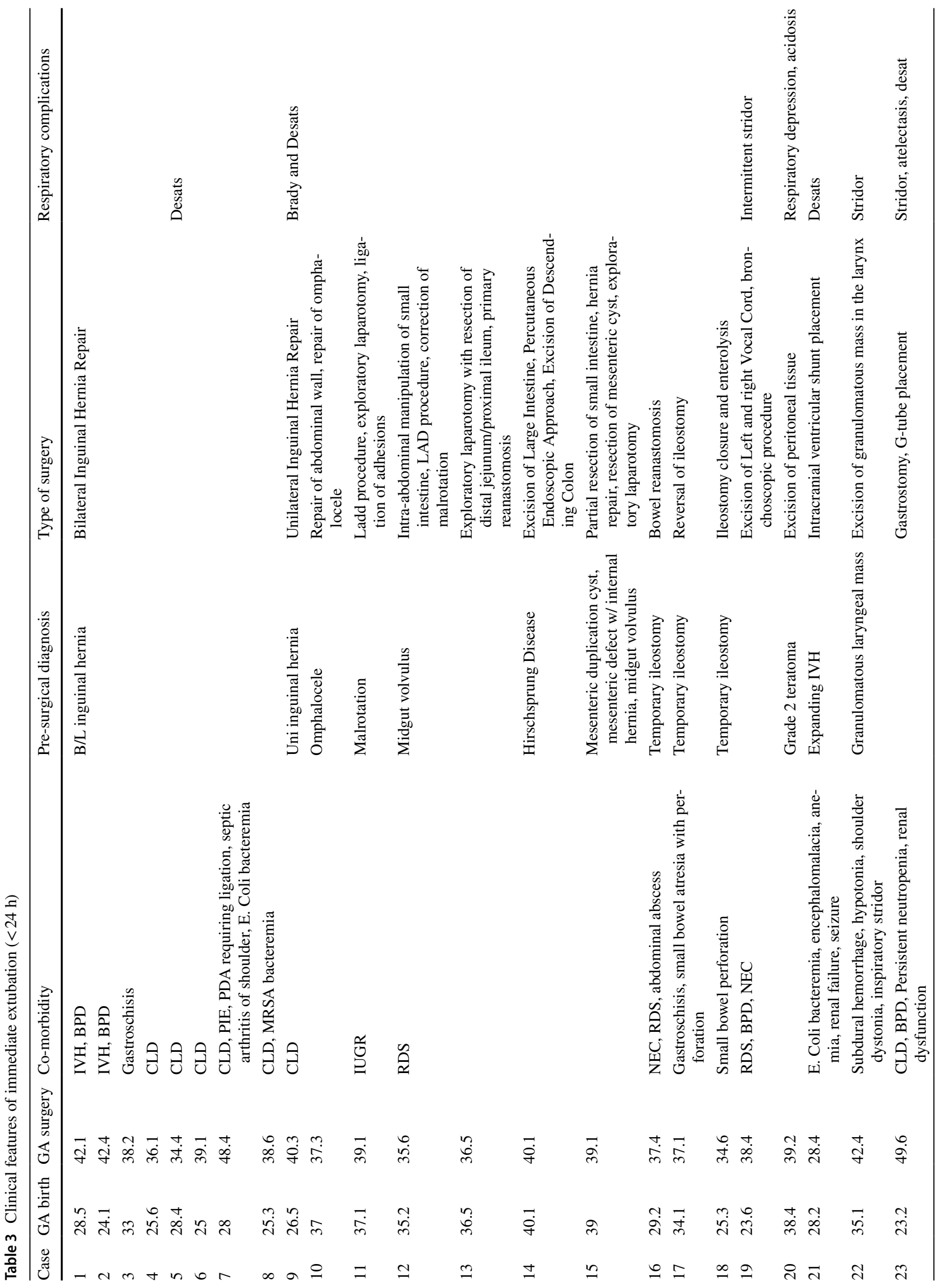



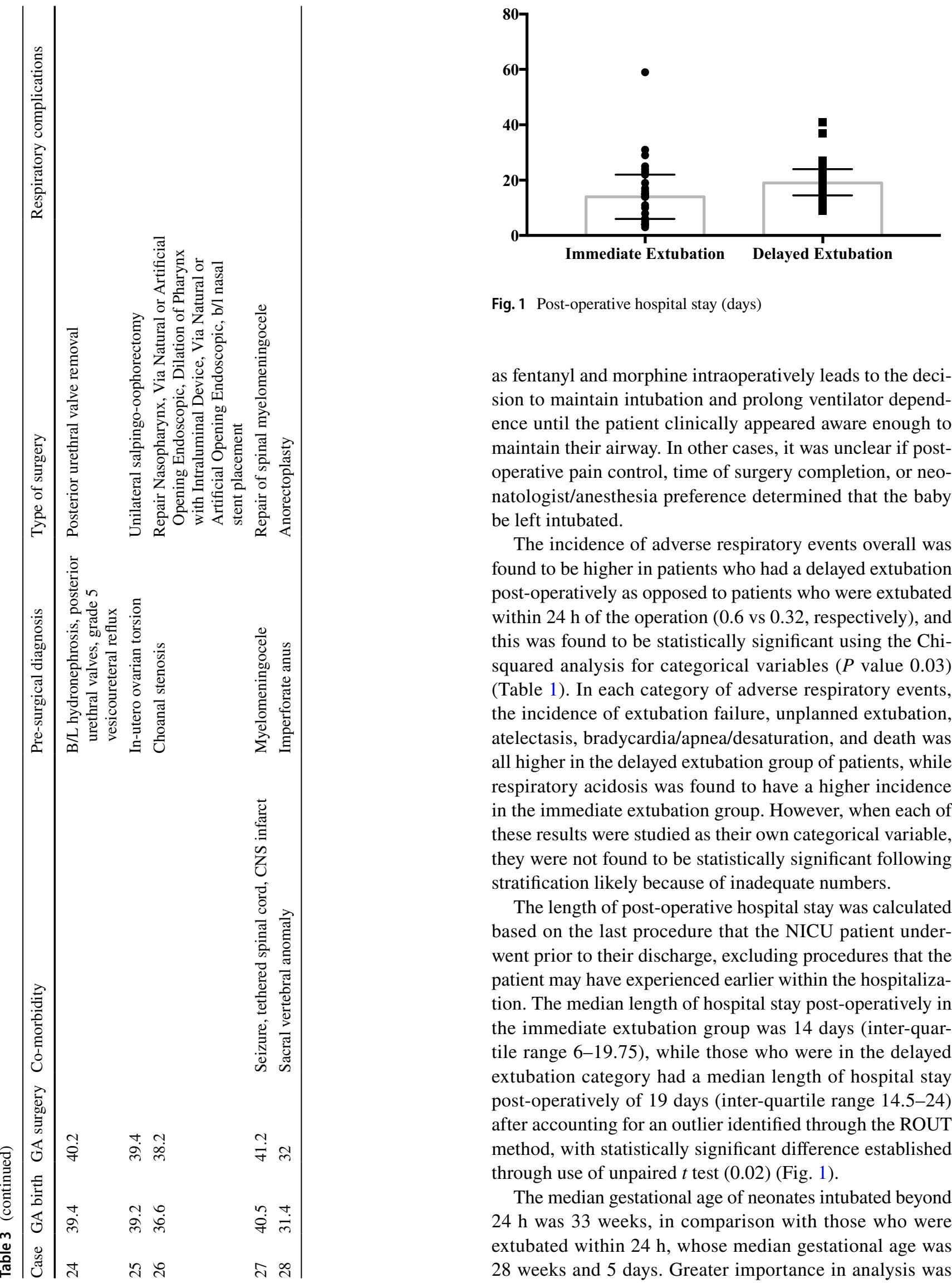

Fig. 1 Post-operative hospital stay (days)

as fentanyl and morphine intraoperatively leads to the decision to maintain intubation and prolong ventilator dependence until the patient clinically appeared aware enough to maintain their airway. In other cases, it was unclear if postoperative pain control, time of surgery completion, or neonatologist/anesthesia preference determined that the baby be left intubated.

The incidence of adverse respiratory events overall was found to be higher in patients who had a delayed extubation post-operatively as opposed to patients who were extubated within $24 \mathrm{~h}$ of the operation ( 0.6 vs 0.32 , respectively), and this was found to be statistically significant using the Chisquared analysis for categorical variables ( $P$ value 0.03 ) (Table 1). In each category of adverse respiratory events, the incidence of extubation failure, unplanned extubation, atelectasis, bradycardia/apnea/desaturation, and death was all higher in the delayed extubation group of patients, while respiratory acidosis was found to have a higher incidence in the immediate extubation group. However, when each of these results were studied as their own categorical variable, they were not found to be statistically significant following stratification likely because of inadequate numbers.

The length of post-operative hospital stay was calculated based on the last procedure that the NICU patient underwent prior to their discharge, excluding procedures that the patient may have experienced earlier within the hospitalization. The median length of hospital stay post-operatively in the immediate extubation group was 14 days (inter-quartile range 6-19.75), while those who were in the delayed extubation category had a median length of hospital stay post-operatively of 19 days (inter-quartile range 14.5-24) after accounting for an outlier identified through the ROUT method, with statistically significant difference established through use of unpaired $t$ test (0.02) (Fig. 1).

The median gestational age of neonates intubated beyond $24 \mathrm{~h}$ was 33 weeks, in comparison with those who were extubated within $24 \mathrm{~h}$, whose median gestational age was 28 weeks and 5 days. Greater importance in analysis was 
accorded to corrected gestational age at surgery as that was considered to be a more important parameter. Corrected gestational age was 36 weeks in those who were extubated beyond $24 \mathrm{~h}$ and 38.5 weeks in those extubated before $24 \mathrm{~h}$ $(P=0.01662)$.

\section{Discussion}

This retrospective study focused on consequences of delaying extubation following a surgical procedure in neonatal patients. The main finding of this study is the significant incidence of overall adverse respiratory events in neonatal surgical patients who were not extubated within $24 \mathrm{~h}$ after surgery. It appears that early post-operative extubation benefits surgical neonates by reducing their respiratory complications. Furthermore, early extubation translates to decrease in length of stay and resultant cost savings for the hospital system. These findings corroborate what has been pointed out by our pediatric cardiac surgery colleagues [1].

Pediatric cardiac surgery leads the way in early extubation protocols [1-3]. The greater coordination between surgeons, pediatric intensive care specialists, and pediatric cardiac anesthesia appears to be the primary driver of quality metrics driven care in that setting. The care for pediatric cardiac surgery patients happens in pediatric cardiac care units (in larger cardiac surgery programs) or in the pediatric intensive care unit (PICU) (in smaller cardiac surgery programs) that are distinct from the NICU and care follows strict protocols. We found that internationally, it is not uncommon for NICUs to not have a written guideline or protocol regarding extubation and weaning steps for their non-cardiac neonatal patients [11].

Aside from a lack of a clear protocol to post-operative extubation, another factor that may lead to the delay in extubation in these patients is the concern for pain management and the effect of pain medications on the respiratory drive. Neonatal patients have a history of having their pain under treated in the past [12-14]. The excessive physiologic stress due to pain after surgical procedures in neonatal patients leads to alteration in stress hormone production and has an adverse homeostatic effect. However, the paradigm of pain management in neonatal patients appears to have shifted too far, where patients are now getting exposed to narcotics and sedatives at an increased rate [15]. An unintended consequence of sedative and narcotic use in intubated neonatal patients is the extension of time that these patients become dependent on mechanical ventilator use [16]. In our study, while we were not able to access pain medication use data due to multiple documentation changes in the hospital system, we did anecdotally notice excessive opioid use in some of these patients that had prolonged intubation times. In the establishment of a post-operative extubation protocol of NICU patients, pain management is a technique that must be addressed to aid transitioning these patients to a smooth and expedited extubation. Developing closer coordination between the surgeon, anesthesiologist, and neonatal intensivist and developing protocols to mimic practice in the pediatric cardiac surgery units will likely improve extubation rates and thereby outcomes.

Enhanced recovery after surgery (ERAS) protocols in adults has lowered complications and facilitated discharge lowering medical costs. While it is not possible to compare the fully developed physiology in adults to that of a preterm infant, we feel that the benefits of enhanced recovery pathways should not be overlooked even in this age group. In our study, the corrected gestational age in the delayed extubation group ( 36 weeks) was lower than in the immediate extubation group (38 weeks). While prematurity may theoretically be an explanation for the delayed extubation, the groups were not statistically different when the hernia population was excluded, suggesting that the two groups were more similar than different. Furthermore, research suggests that even premature patients can be successfully managed by non-invasive respiratory support and re-intubation after failed extubation should not necessarily be deemed as failure $[9,10]$. A duplication of this study in a multicenter prospective setting or a large database retrospective analysis should help shed more light on this important matter.

As seen with this study, in 30 of the 58 identified NICU surgical procedures, patients returned to the NICU while still intubated, and their extubation was dependent on the clinical judgement of the NICU intensivists and the health care team. The clarification of the responsibility and leadership in determining the extubation steps of NICU patients and implementation of a standardized protocol are a possible aspect of medical management that could lead to improvement of care [17]. We believe that developing ERAS protocols for neonates will translate the benefits noted in the adult population to the neonatal population. These findings represent the first report in the non-cardiac neonatal surgery setting of the adverse effects of delayed extubation after surgery and its impact on hospital length of stay. Through an improvement in extubation metrics, we can hope to shorten the course of ICU stay for neonatal surgical patients, possibly decreasing risks of hospital acquired complications, as well as an increased cost efficiency in the NICU department.

The drawbacks of this study are not classifying the operations based on severity of the presenting condition, variable lengths of procedure/anesthesia, having multiple practitioners involved in the care, and the small numbers of cases. However, all of the chosen cohorts were breathing independently prior to the procedure. We feel that perioperative care is an aspect of neonatal care that is neglected and needs greater attention. 


\section{Conclusions}

This study suggests that delaying extubation post-operatively in NICU patients adversely affect the patients' respiratory status that can lead to an increase in length of stay in turn leading to an increased risk of further complications and an increased cost of care. Addressing this critical issue will facilitate advancing the baby along ERAS protocols for other body systems and hopefully lower post-surgical morbidity and cost of care.

Funding None.

\section{Compliance with ethical standards}

Conflict of interest Neither author has a conflict of interest.

Ethical approval The study is retrospective. Study was performed after institutional review board approval and in accordance with the 1964 Helsinki Declaration and its later amendments or comparable ethical standards.

\section{References}

1. Harris KC, Holowachuk S, Pitfield S, Sanatani S, Froese N, Potts JE et al (2014) Should early extubation be the goal for children after congenital cardiac surgery? J Thorac Cardiovasc Surg 148:2642-2648. https://doi.org/10.1016/j.jtcvs.2014.06.093

2. Lawrence EJ, Nguyen K, Morris SA, Hollinger I, Graham DA, Jenkins KJ et al (2013) Economic and safety implications of introducing fast tracking in congenital heart surgery. Circ Cardiovasc Qual Outcomes 6:201-207

3. Howard F, Brown KL, Garside V, Walker I, Elliott MJ (2010) Fast-track pediatric cardiac surgery: the feasibility and benefits of a protocol for uncomplicated cases. Eur J Cardiothorac Surg 37:193-196

4. Gurria J, Kuo P, Kao A, Christensen L, Holterman A (2018) General endotracheal vs. non-endotracheal regional anesthesia for elective inguinal hernia surgery in very preterm neonates: a single institution experience. J Pediatr Surg 52:56-59. https://doi. org/10.1016/j.jpedsurg.2016.10.019

5. Varghese J, Kutty S, Abdullah I, Hall S, Shostrom V, Hammel JM (2016) Preoperative and intraoperative predictive factors of immediate extubation after neonatal cardiac surgery. Ann Thorac Surg 102:1588-1595. https://doi.org/10.1016/j.athoracsur.2016.04.030
6. Wielenga JM, Van den Hoogen A, Van Zanten HA, Helder O, Bol B, Blackwood B (2016) Protocolized versus non-protocolized weaning for reducing the duration of invasive mechanical ventilation in newborn infants. Cochrane Database Syst Rev 3:CD011106. https://doi.org/10.1002/14651858.CD011106.pub2

7. Walsh MC, Morris BH, Wrage LA, Vohr BR, Poole WK, Tyson JE et al (2005) Extremely low birthweight neonates with protracted ventilation: mortality and 18-month neurodevelopmental outcomes. J Pediatr 146:798-804. https://doi.org/10.1016/j.jpeds .2005 .01 .047

8. Miller JD, Carlo WA (2008) Pulmonary complications of mechanical ventilation in neonates. Clin Perinatol 35:273-281. https:// doi.org/10.1016/j.clp.2007.11.004

9. Robbins M, Trittmann J, Martin E, Reber KM, Nelin L, Shepherd E (2015) Early extubation attempts reduce length of stay in extremely preterm infants even if re-intubation is necessary. $\mathbf{J}$ Neonatal Perinatal Med 8:91-97. https://doi.org/10.3233/NPM15814061

10. Biniwale M, Wertheimer F (2017) Decrease in delivery room intubation rates after use of nasal intermittent positive pressure ventilation in the delivery room for resuscitation of very low birth weight infants. Resuscitation 116:33-38. https://doi.org/10.1016/j. resuscitation.2017.05.004

11. Al-Mandari H, Shalish W, Dempsey E, Keszler M, Davis PG, Sant'anna $G$ (2015) International survey on periextubation practices in extremely preterm infants. Arch Dis Child Fetal Neonatal Ed 100:F428-F431. https://doi.org/10.1136/archdischild-2015308549

12. Lago P, Boccuzzo G, Garetti E, Pirelli A, Pieragostini L, Merazzi D et al (2013) Pain management during invasive procedures at Italian NICUs: has anything changed in the last five years? J Matern Neonatal Med 26:303-305. https://doi.org/10.3109/14767 058.2012.733783

13. Menon G, McIntosh N (2008) How should we manage pain in ventilated neonates? Neonatology 93:316-323

14. Anand KJS, Hall RW (2007) Controversies in neonatal pain: an introduction. Semin Perinatol 31:273-274. https://doi. org/10.1053/j.semperi.2007.07.014

15. Prestes ACY, Balda RDCX, Dos Santos GMS, Rugolo LMSDS, Bentlin MR, Magalhães M et al (2016) Painful procedures and analgesia in the NICU: what has changed in the medical perception and practice in a ten-year period? J Pediatr (Rio J) 92:88-95. https://doi.org/10.1016/j.jped.2015.04.009

16. Hall RW (2013) Anesthesia and analgesia in the NICU. NIH Public Access 39:239-254. https://doi.org/10.1016/j.clp.2011.12.013. Anesthesia

17. Fontánez-Nieves TD, Frost M, Anday E, Davis D, Cooperberg D, Carey AJ (2016) Prevention of unplanned extubation in neonates through process standardization. J Perinatol 36:469 